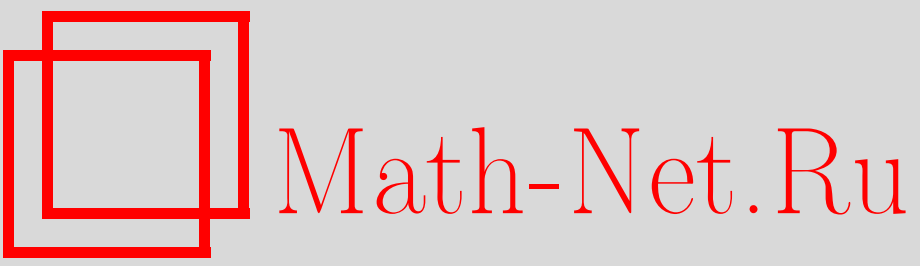

В. И. Питербарг, Б. Стаматович, Грубая асимптотика вероятности одновременных высоких экстремумов двух гауссовских процессов: функционал двойного действия, УМH, 2005, том 60, выпуск 1, 171-172

DOI: https://doi.org/10.4213/rm1398

Использование Общероссийского математического портала Math-Net.Ru подразумевает, что вы прочитали и согласны с пользовательским соглашением

http://www.mathnet.ru/rus/agreement

Параметры загрузки:

IP: 3.95 .254 .165

26 апреля 2023 г., 02:46:29 


\title{
ГРУБАЯ АСИМПТОТИКА ВЕРОЯТНОСТИ ОДНОВРЕМЕННЫХ ВЫСОКИХ ЭКСТРЕМУМОВ ДВУХ ГАУССОВСКИХ ПРОЦЕССОВ: ФУНКЦИОНАЛ ДВОЙНОГО ДЕЙСТВИЯ
}

\author{
В. И. ПИТЕРБАРГ, Б. СТАМАТОВИЧ
}

Рассмотрим два гауссовских процесса $X(t), t \in T$, и $Y(t), t \in S$, с почти наверное ограниченными траекториями, заданные на произвольных множествах $T$ и $S$. Обозначим через $\sigma_{X}^{2}(t)$ и $\sigma_{Y}^{2}(t)$ дисперсии $X$ и $Y$ соответственно, через $r(t, s)$ - взаимную корреляционную функцию процессов $X$ и $Y$ и через $c(t, s)=\frac{\sigma_{X}(t)}{\sigma_{Y}(s)} \wedge \frac{\sigma_{Y}(s)}{\sigma_{X}(t)}-$ функцию мажсорируемости. Для произвольного $D \subset T \times S$ рассмотрим минимум функиионала двойного действия для $X$ и $Y$,

$$
I(X, Y ; D)=\inf _{(t, s) \in D} \frac{1}{\left(\sigma_{X}(t) \vee \sigma_{Y}(s)\right)^{2}}\left(1+\frac{(c(t, s)-r(t, s))^{2}}{1-r(t, s)^{2}} \mathbf{I}_{r(t, s)<c(t, s)}\right) .
$$

Заметим, что в случаях $r= \pm 1$ деление 0 на 0 устранимо.

Теорема 1. Если приведенные выше условия для гауссовских прочессов $X$ и $Y$ выполнены, то для любого подмножества $D \subset T \times S$ имеет место соотношение

$$
\log \mathrm{P}\left(\bigcup_{(t, s) \in D}\{X(t)>u\} \cap\{Y(s)>u\}\right) \sim-\frac{1}{2} I(X, Y ; D) u^{2}
$$

при $и \rightarrow \infty$. В частности,

$$
\log \mathrm{P}\left(\sup _{t \in T} X(t)>u, \sup _{t \in S} Y(t)>u\right) \sim-\frac{1}{2} I(X, Y ; T \times S) u^{2}
$$

npu $u \rightarrow \infty$.

ДОКАЗАТЕЛЬСТВо. Обозначим

$$
P_{2}(u)=\mathrm{P}\left(\bigcup_{(t, s) \in D}\{X(t)>u\} \cap\{Y(s)>u\}\right) .
$$

Из свойств двумерного гауссовского распределения следует, что

$$
\log P_{2}(u) \geqslant \sup _{(t, s) \in D} \log \mathrm{P}(X(t)>u, X(s)>u) \sim-\frac{1}{2} I(X, Y ; D) u^{2} .
$$

Введем функции

$$
\begin{aligned}
\alpha_{X, Y}(t, s) & =1-r(t, s)(r(t, s) \vee c(t, s)), \\
\beta_{X, Y}(t, s) & =r(t, s) \vee c(t, s)-r(t, s),
\end{aligned}
$$

$t, s \in T \times S$. Имеем для $\sigma(t)>0, \sigma(s)>0$ :

$$
\begin{aligned}
\log P_{2}(u) & =\log \mathrm{P}(\exists(t, s) \in D: X(t)>u, X(s)>u) \\
& =\log \mathrm{P}\left(\exists(t, s) \in D: \frac{X(t)}{\sigma(t)}>\frac{u}{\sigma(t)}, \frac{X(s)}{\sigma(s)}>\frac{u}{\sigma(s)}\right) \\
& =\log \mathrm{P}\left(\exists(t, s) \in D: \frac{X(t)}{\sigma(t)}>\frac{u}{\sigma(t) \vee \sigma(s)}, \frac{X(s)}{\sigma(s)}>\frac{c(t, s) u}{\sigma(t) \vee \sigma(s)}\right)
\end{aligned}
$$

Работа первого автора выполнена при поддержке РФФИ (гранты № 04-01-00700, 03-01-00248) и гранта DFG 436 RUS 113/722. 


$$
\begin{aligned}
& =\log \mathrm{P}\left(\exists(t, s) \in D: \frac{\alpha(t, s) X(t)}{\sigma(t)}>\frac{\alpha(t, s) u}{\sigma(t) \vee \sigma(s)}, \frac{\beta(t, s) X(s)}{\sigma(s)}>\frac{\beta(t, s) c(t, s) u}{\sigma(t) \vee \sigma(s)}\right) \\
& \leqslant \log \mathrm{P}\left(\exists(t, s) \in D: \frac{\alpha(t, s) X(t)}{\sigma(t)}+\frac{\beta(t, s) X(s)}{\sigma(s)}>\frac{\alpha(t, s) u}{\sigma(t) \vee \sigma(s)}+\frac{\beta(t, s) c(t, s) u}{\sigma(t) \vee \sigma(s)}\right) \\
& \leqslant \log \mathrm{P}\left(\sup _{(t, s) \in D} \frac{(\sigma(t) \vee \sigma(s))(\alpha(t, s) X(t) / \sigma(t)+\beta(t, s) X(s) / \sigma(s))}{\alpha(t, s)+\beta(t, s) c(t, s)}>u\right) \\
& \sim-\frac{u^{2}}{2}\left(\sup _{(t, s) \in D} \mathrm{E}\left(\frac{(\sigma(t) \vee \sigma(s))(\alpha(t, s) X(t) / \sigma(t)+\beta(t, s) X(s) / \sigma(s))}{\alpha(t, s)+\beta(t, s) c(t, s)}\right)^{2}\right)^{-1} \\
& =-\frac{u^{2}}{2} \inf _{(t, s) \in D} \frac{(\alpha(t, s)+\beta(t, s) c(t, s))^{2}}{(\sigma(t) \vee \sigma(s))^{2} \mathrm{E}(\alpha(t, s) X(t) / \sigma(t)+\beta(t, s) X(s) / \sigma(s))^{2}} \\
& =-\frac{u^{2}}{2} \inf _{(t, s) \in D} \frac{1-2 r(t, s)(r(t, s) \vee c(t, s))+(r(t, s) \vee c(t, s))^{2}}{\left(1-r^{2}(t, s)\right)(\sigma(t) \vee \sigma(s))^{2}} \\
& =-\frac{1}{2} I(X, Y ; D) u^{2}
\end{aligned}
$$

при $u \rightarrow \infty$, где использовался переход от квадранта к касательной полуплоскости, увеличивающий вероятность, и неравенство Ферника [1], из которого следует логарифмическая асимптотика для хвоста распределения максимума. Теорема доказана.

Из цепочки неравенств (5) получаем следующее полезное неравенство для произвольного $D \subset$ $T \times S$,

$$
\begin{aligned}
& \mathrm{P}\left(\bigcup_{t, s \in D}\{X(t)>u\} \cap\{Y(s)>u\}\right) \\
& \quad \leqslant \mathrm{P}\left(\sup _{(t, s) \in D} \frac{\left(\sigma_{X}(t) \vee \sigma_{Y}(s)\right)\left(\alpha_{X, Y}(t, s) X(t) / \sigma_{X}(t)+\beta_{X, Y}(t, s) Y(s) / \sigma_{Y}(s)\right)}{\alpha_{X, Y}(t, s)+\beta_{X, Y}(t, s) c(t, s)}>u\right)_{(6)}
\end{aligned}
$$

которое является логарифммиески асимптотически точньм.

ПримеР. Пусть $X(t), t \in[0, T],-$ гауссовский процесс с нулевым средним и единичной дисперсией. Пусть $P(u, \varepsilon)$ - вероятность существования двух точек $t$ и $s$, разделенных интервалом длины $\varepsilon$ и таких, что $X(t)>u, X(s)>u$. Полагаем $D=\{|t-s| \geqslant \varepsilon\} \cap[0, T]$. В силу теоремы $P(u, \varepsilon) \sim-u^{2} \inf _{D}(1+r(t, s))^{-1}$.

\section{СПИСОК ЛИТЕРАТУРЫ}

[1] X. Fernique // Lecture Notes in Math. 1989. V. 1391. P. 66-73.

Московский государственный университет им. М. В. Ломоносова; Университет Черногории
Представлено А. В. Булинским Принято редколлегией 23.11.2004 